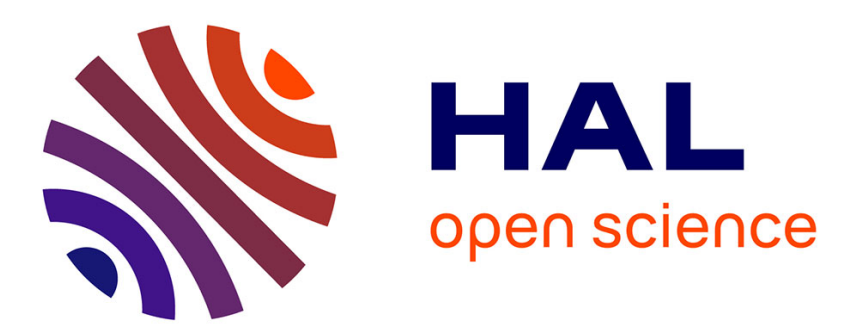

\title{
Real-time monitoring of Yersinia pestis promoter activity by bioluminescence imaging
}

Anne Derbise, Olivier Dussurget, Elisabeth Carniel, Javier Pizarro-Cerdá

\section{To cite this version:}

Anne Derbise, Olivier Dussurget, Elisabeth Carniel, Javier Pizarro-Cerdá. Real-time monitoring of Yersinia pestis promoter activity by bioluminescence imaging. Pathogenic Yersinia Methods and Protocols, 2010, pp.85-97, 2019, Methods in Molecular Biology, 978-1-4939-9541-7. 10.1007/978-14939-9541-7_7. hal-02734553

\section{HAL Id: hal-02734553 \\ https://hal.science/hal-02734553}

Submitted on 2 Jun 2020

HAL is a multi-disciplinary open access archive for the deposit and dissemination of scientific research documents, whether they are published or not. The documents may come from teaching and research institutions in France or abroad, or from public or private research centers.
L'archive ouverte pluridisciplinaire HAL, est destinée au dépôt et à la diffusion de documents scientifiques de niveau recherche, publiés ou non, émanant des établissements d'enseignement et de recherche français ou étrangers, des laboratoires publics ou privés. 
2 Title

3 Real-time monitoring of Yersinia pestis promoter activity by bioluminescence imaging

4 Anne Derbise, Olivier Dussurget, Elisabeth Carniel and Javier Pizarro-Cerdá

6 Running title

$7 \quad$ Real-time monitoring of Yersinia pestis promoter activity

8

\section{Abstract}

10 Bioluminescence imaging has become a major strategy for real-time analysis of dynamic

11 biological processes. In particular, bioluminescent reporter microorganisms have been designed to advance our understanding of infectious diseases. Non-invasive monitoring of light-emitting pathogenic bacteria has revealed novel features of pathogenesis and enabled quantitative and qualitative analysis of antibacterial therapies. Transcriptional gene fusions using the bacterial luciferase operon $\operatorname{lu} x C D A B E$ as a reporter have been successfully used to monitor gene expression in vitro and in vivo, leading to valuable applications and major findings. In this chapter, we describe the construction of Yersinia pestis strains bearing a chromosomal copy of the luxCDABE operon under the control of promoters regulated by temperature and their application to quantify gene expression in real-time in bacteria growing 20 in vitro and in a murine bubonic plague model.

22 Key words Bioluminescence, real-time imaging, reporter system, promoter activity, plague, Yersinia pestis, mice 


\section{Introduction}

27 Bioluminescence is the production and emission of light by a living organism. With the development of highly sensitive photon detectors, in vivo bioluminescence imaging (BLI) techniques have been particularly useful for real-time noninvasive tracking of bacteria within animal models.

Bioluminescent imaging has allowed monitoring of the proliferation and dissemination of Yersinia pestis in real-time in different murine models, such as bubonic and pneumonic plague [1-3]. In these studies, bioluminescence was produced by the Photorhabdus luminescens luciferase which is encoded by the genes $\operatorname{lux} A$ and $\operatorname{lu} B$ that catalyzes the oxidation of a reduced flavin mononucleotide by oxygen leading to the production of photons that can be quantified. The substrate is recycled by the enzymatic complex encoded by genes

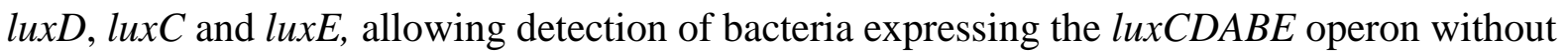
addition of exogenous substrate. The $P$. luminescens luxCDABE operon provides several advantages when expressed in $Y$. pestis. It is compatible with the in vivo mouse body temperature and does not impact fitness of $Y$. pestis. The turnover production and activity of the luciferase A and B subunits allows in vivo multiplication monitoring and correlates with the bacterial load when a constitutive promoter is fused with the lux operon. The short halflife of $P$. luminescens luciferase prevents signal accumulation and makes it appropriate for quantitative studies.

In this chapter, we describe the use of bioluminescence for real-time in vitro and in vivo monitoring of $Y$. pestis promoter activity. To allow the comparative study of gene expression and to avoid side effects from plasmid variable copy numbers and plasmid loss in vivo, we constructed a single chromosomal version of the P. luminescens luxCDABE operon fused downstream of the $Y$. pestis promoter regions of interest. The mini Tn7 system was used for specific chromosomal insertion at the att $\mathrm{Tn} 7$ site between two housekeeping genes, $g \operatorname{lm} S$ and 
51 pstS, encoding glucosamine-6-phosphate synthetase and periplasmic phosphate-binding

52 protein, respectively [4]. Transposition of mini $\operatorname{Tn} 7$ in this intergenic region does not modify

53 the virulence of $Y$. pestis [2].

54 Here we validate and evaluate the sensitivity of the bioluminescent reporter system using the

55 promoters of the caf1M and YPO0499 genes, which are known to be regulated by temperature.

56 The caflM gene is part of the $Y$. pestis F1 capsule encoding operon whose expression is up-

57 regulated at $37^{\circ} \mathrm{C}$, while the gene YPO0499 is part of operon encoding the type VI secretion

58 system whose expression is down-regulated at $37^{\circ} \mathrm{C}$. The constitutively active promoter of

$59 r p l N$, encoding a protein of the 50S ribosomal subunit was used as a control.

60

61 2. Materials

$62 \quad 2.1$ Construction of $Y$. pestis reporter strains

63 1. Fully virulent $Y$. pestis CO92 strain isolated from a fatal human pneumonic plague case [5]

64 (see Note 1).

65 2. Escherichia coli BW19610 strain [6].

66 3. pUC18R6KT mini-Tn7-Km ${ }^{r}$-luxCDABE (Fig. 1) a non-replicative plasmid in Yersinia (see

67 Note 2), which requires a pir expressing strain, such as Escherichia coli BW19610, for its

68 replication.

69 4. Transposase-encoding plasmid pTNS2 [4] (Fig. 1A) (see Note 3).

70 5. Flp recombinase-encoding plasmid pFLP3 [4] (Fig. 1B).

71 6. Thermal Cycler

72 7. Primers for PCR amplification of Y. pestis promoter regions (see Note 4):

$73 \operatorname{PrplN}$, forward 5'-cgcACTAGTtagagtagccaactcgaacataataagc-3' and reverse 5'-

74 ataCCCGGGgctccgctaatgtcaactac-3'; Pcaf1M, forward 5'-cgcACTAGTcttatttggetattcatcc-3' 
and reverse 5'-tgaCCCGGGgcttgctattccttgctattcg-3'; PYPO0499, forward 5'cgcACTAGTcttattttggctattcatcc-3' and reverse 5'-tgaCCCGGGGcgttgctattcettgctattcgct-3'.

8. Primers for promoter sequence verifications: PluxC reverse 5'-cacgaatgtatgtcctgcgtc-3' and PKm forward 5'-tcctatggaactgcctcggt-3'.

9. Primers for PCR verification of mini-Tn7 transposition: PglmS 5'gctatacgtgtttgctgatcaagatg-3', PpstS 5'- acgccaccggaagaaccgatacct-3', PTn7L 5'attagcttacgacgctacaccc-3' and PTn7R 5' - cacagcataactggactgatttc-3'.

10. Qiagen PCR purification kit

11. SpeI and XmaI endonuclease restriction enzymes

12. T4 DNA ligase

13. Sterile distilled water

14. Qiagen Midiprep plasmid purification kit.

15. Lysogeny broth medium (LB): $10 \mathrm{~g}$ tryptone, $5 \mathrm{~g}$ yeast extract and $10 \mathrm{~g}$ of sodium chloride in $1 \mathrm{~L}$ of distilled water.

16. LB agar plates (LBA): $10 \mathrm{~g}$ tryptone, $5 \mathrm{~g}$ yeast extract, $10 \mathrm{~g}$ of sodium chloride and $15 \mathrm{~g}$ of agar in $1 \mathrm{~L}$ of distilled water.

17. LBAH: LBA with $0.002 \%$ (w/v) hemin (see Note 5).

18. LBAHKm: LBAH with kanamycin $(30 \mu \mathrm{g} / \mathrm{mL})$.

19. LBAHCarb: LBAH with carbenicillin $(100 \mu \mathrm{g} / \mathrm{mL})$.

20. LBAHSuc: $10 \mathrm{~g}$ tryptone, $5 \mathrm{~g}$ yeast extract, $100 \mathrm{~g}$ sucrose, and $15 \mathrm{~g}$ of agar in $1 \mathrm{~L}$ of 5 distilled water.

21. Cold, sterile $10 \%$ glycerol.

22. 1X Phosphate buffer saline (PBS).

23. Electroporator

24. Sterile $0.2 \mathrm{~cm}$ electroporation cuvettes. 
101 1. LB

102 2. PBS

103 3. $250 \mathrm{~mL}$ Erlenmeyer flask

104 2. Incubators set to $28^{\circ} \mathrm{C}$ and $37^{\circ} \mathrm{C}$

1053 . Spectrophotometer and cuvettes

106 4. Plate reader capable for photon emission detection at $490 \mathrm{~nm}$.

107 5. 96-well opaque white plate

108

$109 \quad 2.3$ Measurement of promoter activity in a murine bubonic plague model

1101.7 week-old female OF1 mice

111 2. Sterile $26 \mathrm{G} \times 1 / 2 "$ needles and $1 \mathrm{~mL}$ syringes

112 3. Sterile surgical dissection instruments

113 4. Tissue and cell disruption homogenizer (Tissuelyser II, Qiagen)

114 5. Sterile $2 \mathrm{~mL}$ polypropylene microtube with cap filled with 6 to 8 glass beads $(3 \mathrm{~mm}$

115 diameter, Omni international) for disruption of lymph nodes or 2 metal beads (2.4 mm, Omni

116 international) for skin disruption.

117 6. $\mathrm{CO}_{2}$

118 7. Anesthesic mix in PBS: ketamine $20 \mathrm{mg} / \mathrm{mL}$ (Imalgene 1000, Bayer) and xylazine $2 \mathrm{mg} / \mathrm{mL}$

119 (Rompun 2\%, Merial).

120 8. Poly(methyl methacrylate) confinement box (TEM SEGA) (see Note 6).

121 9. Imaging System (IVIS Spectrum, Perkin Elmer)

122 10. Living Image 4.3.1 software (Caliper Life Sciences)

\section{Methods}




\subsection{Promoter amplification and cloning to generate a bioluminescent reporter plasmid}

127 (Fig. 1A)

128 1. Amplify the promoter region of interest using standard PCR methods with forward and

129 reverse primers for $\mathrm{P}^{r p l N}, \mathrm{P}^{c a f l M}$ and $\mathrm{P}^{Y P O 0499}$ regions.

130 2. Purify PCR products using the Qiagen PCR purification kit.

131 3. Digest the purified PCR product and the pUC18R6KT mini-Tn7-Km ${ }^{r}-l u x C D A B E-$ based

132 vector with SpeI and XmaI following the enzyme manufacturer's instructions.

133 4. Ligate the digested PCR product and plasmid following the manufacturer's instructions 134 with T4 DNA ligase overnight at $16^{\circ} \mathrm{C}$.

135 5. Electroporate 1-10 $\mu \mathrm{L}$ of the ligation reaction into Escherichia coli BW19610

136 electrocompetent cells [7]. Add $1 \mathrm{~mL}$ of $\mathrm{LB}$ and incubate at $37^{\circ} \mathrm{C}$ for $45 \mathrm{~min}$. Plate various

137 bacterial suspensions (pure to $1 / 100$ dilution) on LBA supplemented with $100 \mu \mathrm{g} / \mathrm{mL}$

138 carbenicillin and incubate overnight at $37^{\circ} \mathrm{C}$.

139 6. Check the correct insertion and sequence of the promoter of interest by restriction enzyme

140 digestion using SpeI and XmaI and by sequencing using primers PKm and PluxC. The

141 recombinant plasmid will be referred in this chapter as pUC18-mini-Tn7-K-Pestis -lux.

$143 \quad 3.2$ Construction of a single-copy bioluminescent reporter gene expression system in $Y$.

144 pestis (Fig. 1)

145 1. Purify plasmids pTNS2 (transposase provider) and pUC18-mini-Tn7-K- $P^{\text {pestis }}$-lux using the

146 Qiagen Midiprep plasmid purification kit.

147 2. Prepare electrocompetent $Y$. pestis CO92 cells resuspended in ice cold $10 \%$ glycerol (see $148 \quad$ Note 7). 
149 3. Electroporate $50 \mu \mathrm{L}$ of electrocompetent $Y$. pestis cells with $400 \mathrm{ng}$ of each plasmid using 150 the following settings: $2.5 \mathrm{kV}, 200 \Omega$ and $25 \mu \mathrm{F}$.

151 4. Add $1 \mathrm{~mL}$ of $\mathrm{LB}$ and incubate at $28^{\circ} \mathrm{C}$ under agitation for $90 \mathrm{~min}$.

152 5. Plate various dilutions on LBAHKm and incubate for $48 \mathrm{~h}$ at $28^{\circ} \mathrm{C}$ (recommended dilutions, 153 $1 / 10$ to $\left.1 / 10^{3}\right)$.

6. Verify the correct insertion of mini-Tn7-K- $P^{\text {pestis-lux }}$ (Fig. 1) into the chromosomal glmSpstS intergenic region by PCR using the pairs of primers PglmS/PTn7R, PpstS/PTn7L and PglmS/ PpstS flanking the att Tn7 insertion site (see Note 8).

157 7. Prepare $Y$. pestis CO92:: mini-Tn7-K- $\mathrm{P}^{\text {pestis }}$-lux electrocompetent cells resuspended in ice 158 cold $10 \%$ glycerol (see Note 7).

1598 . Remove the kanamycin resistant cassette by electroporation of $50 \mu \mathrm{L}$ electrocompetent $Y$.

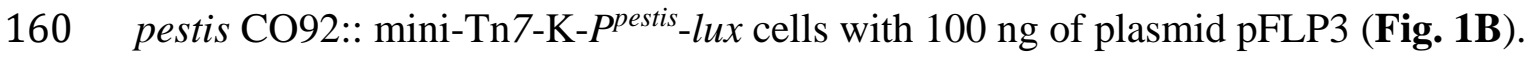
9. Select the recombinant pFLP3 harboring cells by plating various dilutions on LBAHCarb and incubate $48 \mathrm{~h}$ at $28^{\circ} \mathrm{C}$. 10. Replicate plate individual colonies on both LBAHKm and LBAH plates to identify the 164 clones that have lost the kanamycin resistance gene.

165 11. Eliminate the pFLP3 plasmid by growing Y. pestis CO92::mini-Tn7-P ${ }^{\text {pestis }}$-lux on LB 166 overnight at $28^{\circ} \mathrm{C}$.

167 12. Serial dilute the overnight culture and plate $100 \mathrm{ul}$ of $1 / 100$ to $1 / 10^{6}$ dilutions on 168 LBAHSuc plates.

169 11. Replicate plate individual colonies on both LBAHCarb and LBAH to identify the clones 170 that have lost pFLP3.

171 12. Check for the presence of known unstable $Y$. pestis virulence genetic elements such as 172 plasmids, pYV and pPla and the High Pathogenicity Island by PCR [8] (see Note 9). 


\subsection{In Vitro detection of promoter activity}

175 1. Make a lawn of $Y$. pestis recombinant strain (CO92 :: mini-Tn7-K- $P^{\text {pestis }}$-lux) by spreading 176 an aliquot from a $-80^{\circ} \mathrm{C}$ frozen stock and incubating overnight at $28^{\circ} \mathrm{C}$ on $\mathrm{LBAH}$.

177 2. Resuspend bacterial cells in fresh $10 \mathrm{~mL} \mathrm{LB}$ by scraping from the bacterial lawn, adjust 178 optical density to 1 , dilute $1 / 100$ in $50 \mathrm{~mL} \mathrm{LB}$ and grow under constant agitation (see Note 179 10). In this study two temperatures were tested, $28^{\circ} \mathrm{C}$ and $37^{\circ} \mathrm{C}$ (Fig. 2).

180 3. Measure the optical density at $600 \mathrm{~nm}$ over time using $1 \mathrm{~mL}$ samples (Fig. 2) 4. Measure the photon emission per second (RLU) over time by transfering $0.1 \mathrm{~mL}$ sample from the $50 \mathrm{~mL}$ growth in a 96 well plate (Fig. 2) (see Note 11).

5. To determine promoter activity, divide the number of photon/sec (RLU) by the absorbance at $600 \mathrm{~nm}$ in exponential phase to correct for the relative differences in cell number between 185 strains.

186

\subsection{In vivo detection of $Y$. pestis promoter activity in a murine bubonic plague model}

188 Animal experiments must receive approval from national and institutional committees.

189 Our protocol was approved by the French committee CETEA $\left(\mathrm{n}^{\circ} 2014-0025\right)$ and Institut 190 Pasteur CHSCT ( $\left.{ }^{\circ} 0399\right)$.

191 1. Grow bioluminescent $Y$. pestis on $\mathrm{LBAH}$ plates overnight at $28^{\circ} \mathrm{C}$, as described in section $192 \quad 3.3 .1$.

193 2. Resuspend bacteria in PBS and adjust OD600 to 0.1, as described in section 3.3.2.

194 3. Prepare serial dilutions in PBS.

195 4. Inject the required amounts of bacterial cells within a maximal volume of $50 \mu \mathrm{L}$ for 196 subcutaneous injection (see Note 12). In the experiment shown in figure 3, 10e5 CFU were 197 injected (see Note 13) 
198 5. At desired time points, anesthetize the mice by injecting intraperitoneally $100 \mu \mathrm{l}$ of the

199 anesthetic mix (ketamine/xylazine).

200 6. Place mice in a confinement box (see Note 6).

201 7. Place the confinement box into the IVIS imaging chamber with the mice injection site

202 facing the CCD camera.

203 8. Choose the field of view according to the number of animals to be imaged (see Note 14).

2049 . Set up the imaging acquisition conditions on the control panel by selecting luminescent

205 imaging mode, block excitation filter and open emission filter.

206 10. Adjust the binning to "small" and the exposure time according to the intensity of the

207 signal (between $5 \mathrm{sec}$ to 2 min exposure time) (see Note 15).

208 11. Click "acquire" to measure luminescence (Fig. 3).

209 12. Use the tool palette/image adjust to adjust the color scale in order to visualize all sites that 210 exhibit signals above the background (see Note 16).

211 13. Create regions of interest (ROI) by using the ROI tool (see Note 17) and measure ROIs.

212 14. In order to evaluate the background bioluminescent signal, measure the number of

213 photons emitted from the same ROI of a non-infected mouse placed in the same confinement

214 box as infected mice (see Note 16).

215 15. Calculate the luminescence to determine changes in luciferase activity over time.

216 Promoter activity is measured by counting the photons emitted by the bacteria. To allow

217 comparisons between signals express the ROI bioluminescence in average radiance

218 (photons $/ \mathrm{sec} / \mathrm{cm}^{2} /$ steradian).

219 13. To determine the number of bacteria in the infected tissues, euthanize mice with $\mathrm{CO}_{2}$.

220 14. Harvest the tissues aseptically and place them in a $0.5 \mathrm{~mL}$ sterile PBS tubes containing

221 glass or metal beads. 
15. Apply a 2 min tissue disruption cycle of 30 agitations/sec. with the TissueLyser II Qiagen

223 apparatus (see Note 18)

224 16. Spread $50 \mu \mathrm{L}$ of serial dilutions of the cell suspension on LBAH plates and incubate $48 \mathrm{~h}$ 225 at $28^{\circ} \mathrm{C}$ for colony counts.

226 17. Results are given in ROI per CFU (Fig. 3B).

4. Notes

1. Experiments performed on fully virulent $Y$. pestis require a Biosafety Level 3 laboratory and animal facility.

2. The plasmid pUC18R6KT mini-Tn7-Km ${ }^{r}$-luxCDABE was constructed by subcloning (i) the

$232 \mathrm{Km}$ resistance cassette from $\left(\mathrm{Km}^{\mathrm{r}}\right)$ pFKM1[4] into the SacI restriction site and (ii) the

P. luminescens luxCDABE operon into the ApaI/XmaI restriction sites of pUC18R6KT mini-

$\operatorname{Tn} 7$ [4]. The resulting plasmid does not contain any promoter region for luxCDABE expression and is used for evaluation of bioluminescence background in in vitro studies, as well as for subsequent cloning of the different $Y$. pestis promoters to be studied.

3. Plasmid pTNS2 is not replicative in $Y$. pestis. Efficient production of the transposase immediately after pTNS2 introduction in $Y$. pestis allows transposition of the mini Tn7. Loss of the transposase encoding plasmid will prevent subsequent transposition of $\operatorname{Tn} 7$.

240 Verification of the loss of pTNS2 after Tn7 transposition in $Y$. pestis chromosome is

241 performed by testing carbenicillin susceptibility on LBAHCarb.

242 4. For a directional cloning of promoter regions upstream of the $l u x C D A B E$ operon, forward 243 and reverse primers contain 5' SpeI and XmaI restriction sites, respectively (Fig. 1A).

244 5. When colony forming units (CFU) count have to be performed on Y.pestis suspension it is 245 recommended to supplement the agar lysogeny broth medium with $0.002 \%(\mathrm{w} / \mathrm{v})$ porcine 246 hemin. The presence of hemin in the medium will allow individual bacteria to grow and form 
247 a CFU within 48 hours at $28^{\circ} \mathrm{C}$. If hemin is omitted colonies will be small, difficult to count

248 and the number of colonies could be underestimated.

249 6. When animals are infected with a class 3 pathogen, their transportation and visualization in 250 the IVIS spectrum system should be conducted in a biosafety containment box. In our study

251 we used a Poly(methyl methacrylate) confinement box (TEM SEGA) to warrant the

252 confinement of infected animals and to allow the photon acquisition by the CCD detector.

253 7. To prepare electrocompetent $Y$. pestis, bacteria were grown overnight at $28^{\circ} \mathrm{C}$ in $\mathrm{LB}$. The 254 culture was diluted (1/50) in fresh broth and grown until reaching A600=0.8. Bacterial cells 255 were harvested by centrifugation $\left(4000 \mathrm{xg}, 15 \mathrm{~min}, 4^{\circ} \mathrm{C}\right)$, washed twice in cold sterile distilled 256 water and once in $10 \%$ glycerol. The pellet was resuspended in $10 \%$ glycerol to reach a 257 concentration factor of 500 times compared to the original growth volume. Electrocompetent 258 cells were aliquoted in $50 \square \mathrm{L}$ and frozen at $-80^{\circ} \mathrm{C}$ until use.

259 8. Correct insertion of the mini-Tn7 transposon is verified by the amplification of $770 \mathrm{bp}$ 260 and 336 bp PCR fragments with primers PpstS/PTn7L and PglmS/ PpstS respectively and 261 absence of amplification with primers $g \operatorname{lm} S$ - $p s t S$ when the following standard PCR program 262 is used: 1 cycle at $95^{\circ} \mathrm{C}$ for $3 \mathrm{~min}, 35$ cycles (i) at $95^{\circ} \mathrm{C}$ for $30 \mathrm{sec}$, (ii) at $55^{\circ} \mathrm{C}$ for $30 \mathrm{sec}$, 263 and (iii) at $72^{\circ} \mathrm{C}$ for $1 \mathrm{~min}$ and finally 1 cycle at $72^{\circ} \mathrm{C}$ for $3 \mathrm{~min}$.

264 9. Presence of the pYV and pPla plasmids and the high pathogenicity island are verified by 265 PCR amplifications of 565 bp yopM, 480 bp pla and 434 bp irp2 fragments using primer pairs 266 YopM-f (5'-ataactcatcgggggcaaaat-3')/YopM-r (5'-gcgttatttatccgaatttagc-3'), pla-f (5'267 atcttactttccgtgagaag-3')/pla-r (5'-cttggatgttgagcttccta-3') and irp2-f (5'-tgatgagatggttacactgg268 3')/irp2-r (5'-gtcgttatggcgtaaatcat-3'), respectively. The standard PCR program indicated in 269 Note 8 is used.

270 10. Conditions of growth culture may vary depending on the known or putative activity of the 271 promoter region studied. 
11. Since $Y$. pestis tends to aggregate when grown in LB, we recommend to perform the in

273 vitro growth in a large volume $(50 \mathrm{~mL} \mathrm{LB})$ in a $250 \mathrm{~mL}$ Erlenmeyer flask under $140 \mathrm{rpm}$

274 agitation and measure the absorbance overtime by taking $0.1 \mathrm{~mL}$ samples.

275 12. To facilitate the subcutaneous injection, the fur of the animal can be locally shaved $24 \mathrm{~h}$

276 before the injection. Depending the site of injection anesthesia can be required prior to the

277 injection. In the experiment shown in figure 3 the animals were not anesthetized.

278 13. The volume and dose injected can vary depending on the injection mode, site and the 279 expected level of signal at early time points of the infection.

280 14. IVIS spectrum allows the use of different fields of view. When more than three animals

281 are imaged use field of view D. Sensitivity of the detection camera may vary depending on

282 the field of view chosen during acquisition. If a comparative study is performed make sure to

283 use the same field of view throughout the experiment.

284 15. For quantification, make sure to use an exposure time that does not lead to signal

285 saturation. In the image display, a message indicates saturated signals. In most of our

286 experiments the exposure time varied from 5 to $240 \mathrm{sec}$ with a small binning. If a weak signal

287 is expected, the exposure time can be increased up to 5 min with a large binning.

288 16. When several infected animals are to be imaged within the same containment box, it is 289 important to evaluate the level of the background signal by imaging a non-infected animal 290 hosted in the same box. This level may change depending on the intensity of the overall signal 291 within the same box.

292 17. The shape and size of the ROI will be adjusted to the shape and size of the site exhibiting 293 a bioluminescent signal. In the case of the site of injection shown in figure 3 , the shape and 294 size of the ROI were drawn by using the function "duplicate".

295 18. For tissue disruption, other homogenizer apparatuses can be used. If so, validation of the 296 efficiency of bacterial count must be performed. 


\section{Acknowledgements}

299 We thank Marie-Anne Nicola for her advice in the use of the bioluminescence system at the

300 Photonic BioImaging facility of the Institut Pasteur.

$301 \quad$ References

302 1. Nham $\mathrm{T}$ et al (2012) Imaging of bubonic plague dynamics by in vivo tracking of 303 bioluminescent Yersinia pestis. PLoS One 7(4): e34714

3042 . Sun Y et al (2012) Development of bioluminescent bioreporters for in vitro and in $305 \quad$ vivo tracking of Yersinia pestis. PLoS One 7(10): e47123

306 3. Gonzalez RJ et al (2012) Bioluminescence imaging to track bacterial dissemination of $307 \quad$ Yersinia pestis using different routes of infection in mice. BMC Microbiol 12(147

308 4. Choi KH et al (2005) A Tn7-based broad-range bacterial cloning and expression $309 \quad$ system. Nat Methods 2(6): 443-448

310 5. Doll JM et al (1994) Cat-transmitted fatal pneumonic plague in a person who traveled from Colorado to Arizona. Am J Trop Med Hyg 51(1): 109-114

312 6. Metcalf WW, W Jiang, and BL Wanner (1994) Use of the rep technique for allele replacement to construct new Escherichia coli hosts for maintenance of R6K gamma origin plasmids at different copy numbers. Gene 138(1-2): 1-7

315 7. Dower WJ, JF Miller, and CW Ragsdale (1988) High efficiency transformation of $E$. coli by high voltage electroporation. Nucleic Acids Res 16(13): 6127-6145

317 8. Tsukano H et al (1996) Detection and identification of Yersinia pestis by polymerase chain reaction (PCR) using multiplex primers. Microbiol Immunol 40(10): 773-775 
322 Fig. 1 Construction of the bioluminescent reporter vector to study $Y$. pestis promoter activity.

323 (A) Suicide delivery pUCR6K-mini Tn7 based vector. Tn7L and Tn7R, left and right ends of

$324 \mathrm{Tn} 7 ; \mathrm{T}_{0} \mathrm{~T}_{1}$, transcriptional terminators; bla, $\beta$-lactamase-encoding gene; $\mathrm{Km}^{\mathrm{R}}$ kanamycin 325 resistance encoding cassette flanked by FRT, Flp recombinase target; $\mathrm{P}^{\text {pestis }}$, Y. pestis 326 promoter regions; luxCDABE, Photorabdus luminescens lux operon where luxA and luxB 327 encode the subunits $\mathrm{A}$ and $\mathrm{B}$ of bacterial luciferase, while $\operatorname{lux} C$, $\operatorname{lux} D$, and $\operatorname{lux} E$ encode the 328 subunits of a fatty acid reductase that provides the fatty aldehydes to the luciferase. Only 329 enzyme restriction sites used for cloning $\mathrm{Km}^{\mathrm{R}}$, promoter regions and $l u x$ operon are indicated. 330 pTNS2, helper suicide plasmid encoding the specific TnsABC+D transposition pathway. 331 Transposition of mini $\mathrm{Tn} 7-\mathrm{Km}^{\mathrm{R}}-\mathrm{P}^{\text {pestis }}$-lux into $Y$. pestis chromosome is achieved by co332 electroporation of competent $Y$. pestis CO92 cells with pTNS2 and pUCR6K-KmR-lux. $333 a t t \operatorname{Tn} 7$ unique insertion site located in the 482-bp $g \operatorname{lm} S$ - $p$ st $S$ intergenic region.

334 (B) Removal of the $\mathrm{Km}^{\mathrm{R}}$ cassette. Mini $\mathrm{Tn} 7-\mathrm{Km}^{\mathrm{R}}-\mathrm{P}^{\text {pestis }}$-lux reporter sytem integrated as a 335 monocopy in $Y$. pestis chromosome; pFLP3, Flp recombinase encoding plasmid. Plasmid 336 pFLP3 is electroporated into the bioluminescent $Y$. pestis CO92 recombinant strain to remove 337 the KmR cassette in order to avoid any transcriptional interference with the bioluminescence 338 reporter system.

340 Fig. 2 Detection of bioluminescence in vitro.

341 Yersinia pestis CO92 recombinant strains were pre-grown on LBAH plate. Then bacterial 342 cells were resuspended in PBS, optical density at $600 \mathrm{~nm}$ was adjusted and a $50 \mathrm{~mL}$ LB flask 343 was inoculated 1/100e to obtain approximately $10 \mathrm{e} 7 \mathrm{CFU} / \mathrm{mL}$ and incubated under agitation. 344 Optical density and photon emission were recorded overtime by taking a $1 \mathrm{~mL}$ and $0.1 \mathrm{~mL}$ 345 aliquot of the growth suspension, respectively. (A) Detection of bioluminescence signals 346 obtained with strains CO2:: Tn7- $\mathrm{P}^{\text {less }}-l u x$ and $\mathrm{Tn} 7-\mathrm{P}^{r p l N}-l u x$ when grown in $\mathrm{LB}$ at $28^{\circ} \mathrm{C}$ and 
$37^{\circ} \mathrm{C}$. The CO92::Tn7-P ${ }^{\text {less }}$-lux strain was used as a negative control to evaluate in each

348 growth condition the bioluminescence background. The promoter region of the $r p l N$

349 ribosomal encoding gene was used as a constitutively active promoter in each growth

350 condition. Since the ratio RLU over CFU did not vary significantly over time nor with

351 temperature for the two control strains, only an average of the signals measured in each

352 growth condition are indicated. Detection of $Y$. pestis $\mathrm{P}^{c a f}(\mathrm{~B})$ and $\mathrm{P}^{Y P O 0499}$ (C) activities, two

353 temperature sensitive promoters in $\mathrm{LB}$ at $28^{\circ} \mathrm{C}$ and $37^{\circ} \mathrm{C}$. The increase of bioluminescence

354 reflects the activity of the $\mathrm{P}^{c a f}$ promoter showing upregulated $Y$. pestis capsule expression,

355 while the decrease of bioluminescence reflects the downregulated expression of the type VI

356 secretion YPO0499 gene at $37^{\circ} \mathrm{C}$. Statistical analyses were performed with an unpaired t-test

$357(\mathrm{p}<0,0001 * * * *, \mathrm{p}<0,0003 * * *, \mathrm{p}<0,001 * *)$ using Prism 5 GraphPad software.

358

359 Fig. 3 Bioluminescence imaging of $Y$. pestis infected mice.

360 (A) OF1 mice were infected subcutaneously in the linea alba with 10e5 CFU of $Y$. pestis

361 CO92::Tn7-P ${ }^{r p l N}-l u x$ or $\mathrm{P}^{Y P O 0499}$-lux and imaged at different time points using an IVIS

362 Spectrum imaging system. Sequential images from representative animals are shown. The

363 color scales represent the light emission and is kept constant throughout the observation

364 period. In this study a small binning and an acquisition time of 10 s to 240 s were used.

365 Uninfected mice (UI) were used to set the light emission background. Regions of interest

366 (ROI) were drawn using the Living Image 4.3.1 software and average bioluminescence

367 (photon/sec/cm2/sr) was calculated for the injection site, as shown here for the $\mathrm{P}^{r p l N}$-fusion at

$3680.5 \mathrm{~h}$ post injection in the skin. Bioluminescence signal is detected in the skin at the site of

369 injection. The increased signal observed with the $\mathrm{P}^{r p l N}$-lux fusion construct reflects $Y$. pestis

370 multiplication. In addition, bacterial dissemination to the inguinal and axillary draining lymph

371 nodes is detected $44 \mathrm{~h}$ post infection. 
(B) Evaluation of promoter activity during $Y$. pestis multiplication in the skin of OF1 mice.

373 Mice were sacrificed at indicated time points. The site of injection was dissected and the skin 374 was homogenized to determine bacterial loads (CFU) and compare them to the measured ROI 375 (photon/sec/cm2/sr). $\mathrm{P}^{r p l N}-l u x$ fusion illustrates the constitutive expression of $r p l N$, where the 376 ROI/CFU ratio is similar over the time. The $\mathrm{P}^{Y P O 0499}$-lux fusion demonstrates the decrease of 377 YPO0499 expression when the pathogen multiplies in the skin as exemplified by the decrease 378 ROI/CFU ratio.

379 
$415 \quad$ Figure 1

A

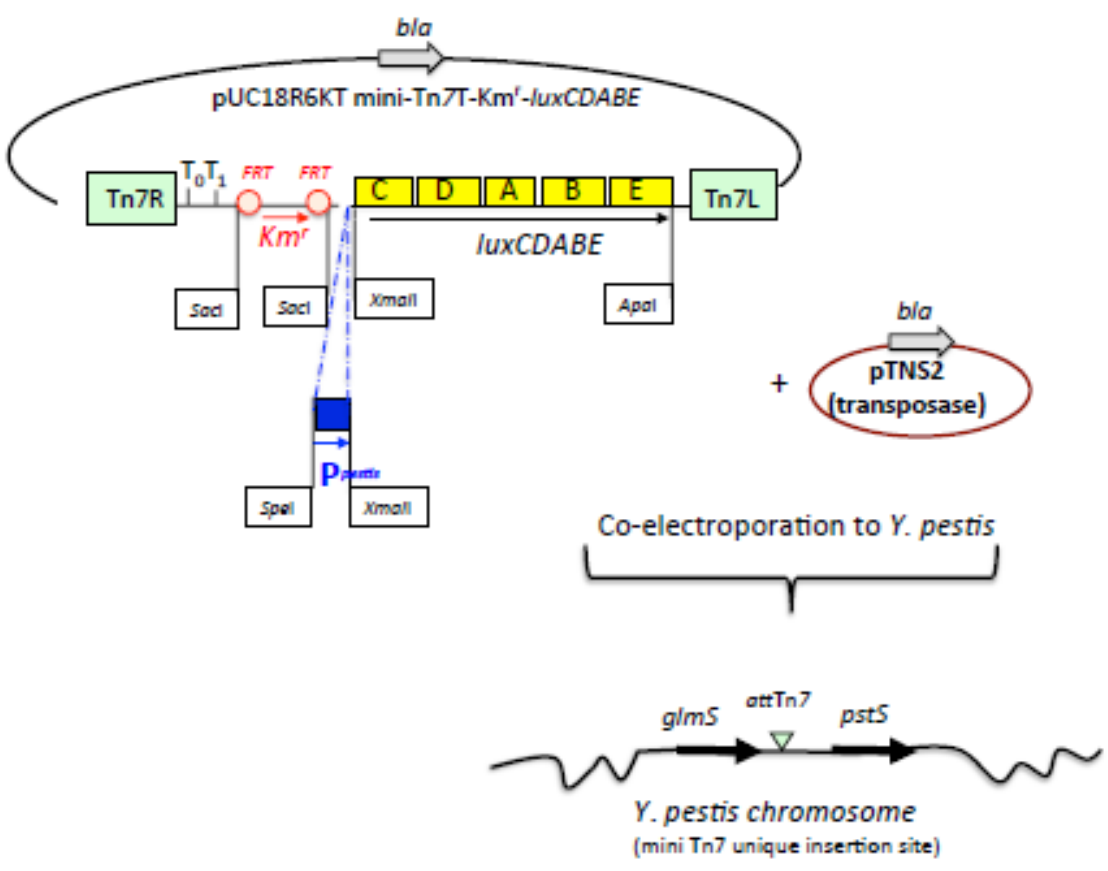

B

Single chromosomal copy of the lux operon under the control of $Y$. pestis promoter

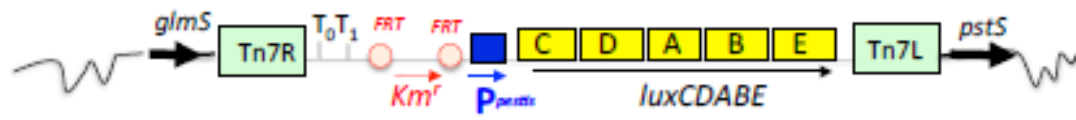

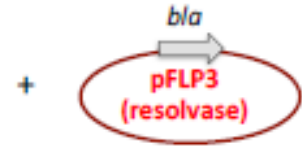

Removal of the $\mathrm{Km}$ resistance cassette

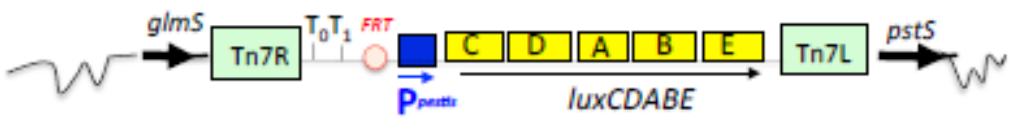


418 Figure 2
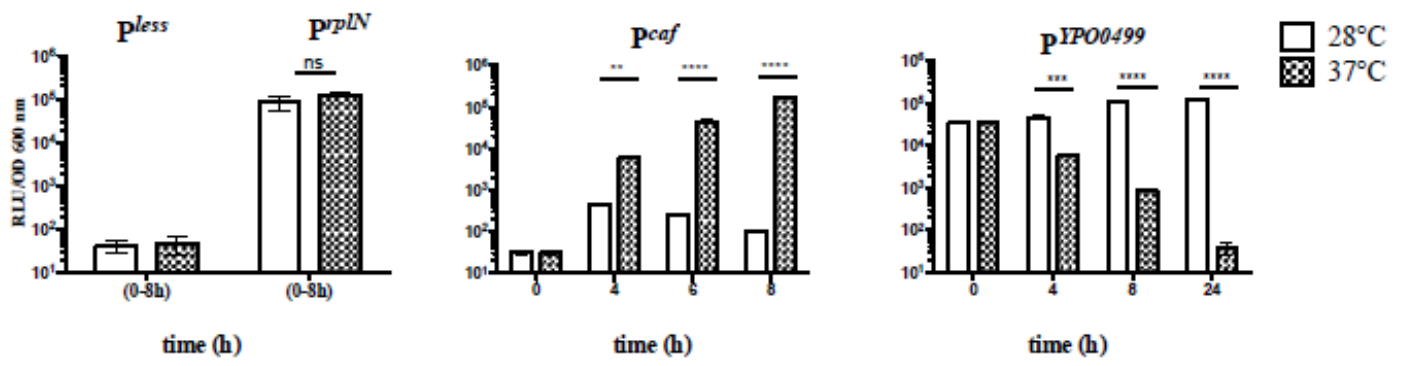

419

420

421

422

423

424

425

426

427

428

429

430

431

432

433

434

435

436 
$437 \quad$ Figure 3

A

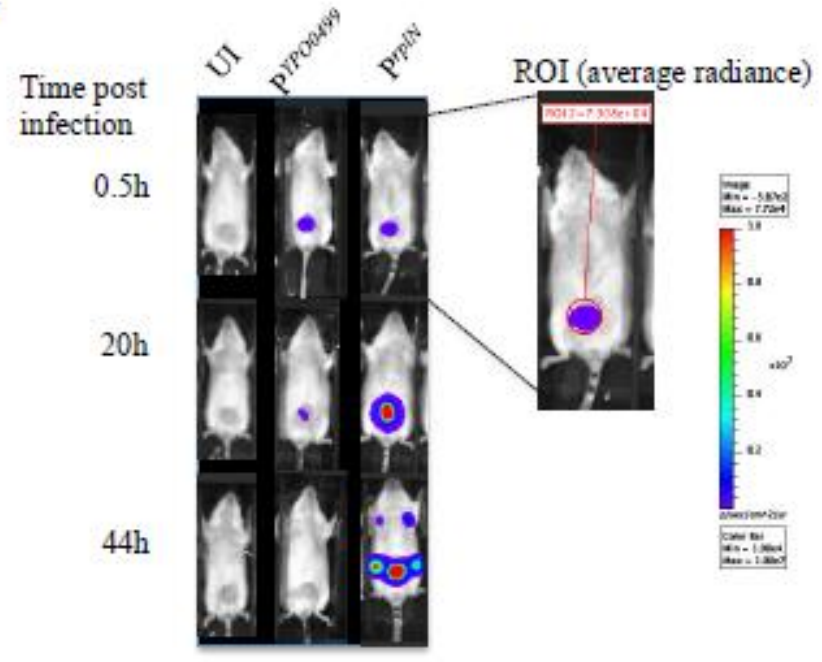

B

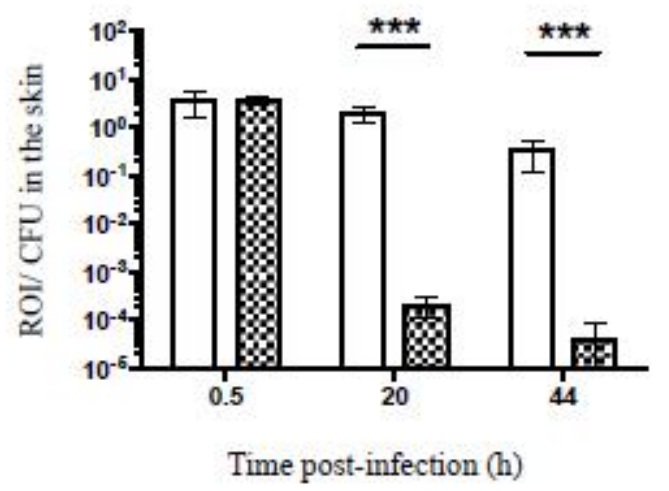

438 